\title{
The Tooth Bandaid - Restoring Esthetics
}

\author{
Siya Dukle ${ }^{1}$, Alok Patel $^{2}$, Sanket Kunte ${ }^{3}$, Shweta Jajoo ${ }^{4}$ \\ ${ }^{I}$ (Post Graduate student, Department of Pedodontics and Preventive Dentistry, Bharati Vidyapeeth Deemed \\ University Dental College and Hospital, Pune, India) \\ ${ }^{2}$ (Head of Department, Department of Pedodontics and Preventive Dentistry, Bharati Vidyapeeth Deemed \\ University Dental College and Hospital, Pune, India) \\ 3(Associate Professor, Department of Pedodontics and Preventive Dentistry, Bharati Vidyapeeth Deemed \\ University Dental College and Hospital, Pune, India) \\ 4(Assistant Professor, Department of Pedodontics and Preventive Dentistry, Bharati Vidyapeeth Deemed \\ University Dental College and Hospital, Pune, India)
}

\begin{abstract}
Trauma to the anterior teeth are a common sight these days, affecting children and adolescents. Coronal tooth fractures are frequently encountered. When the tooth fragment is available, reattachment of fractured tooth fragments can be a good treatment alternative which provides good and long-lasting esthetics as the tooth's original anatomic form, color, wear resistance, morphology and translucency in the restoration is maintained. It also restores function, provides a positive psychological response, and is a relatively simple procedure.. This article reports a coronal tooth fracture case that was successfully treated using tooth fragment reattachment.
\end{abstract}

Keywords: esthetics,fragment reattachment, restorations, trauma

\section{Introduction}

Trauma to the anterior teeth is common in children and adolescents. Most dental injuries occur between 2 and 3 years and between 8 and 12 years of age, and are more common in boys than in girls because of their active involvement in extracurricular activities [1]. The most frequent causes of trauma are falls, bicycle accidents, sports activities, collision with other people and objects, and domestic violence fights and physical assault [2]. Prevalence of trauma to maxillary incisors accounts for about $37 \%$ due to their anterior positioning and protrusion caused by the eruptive pattern[3]. Coronal fracture is the frequent type of dental trauma in the permanent dentition. Eighty percent of traumatized incisors have fracture lines proceeding in an oblique direction from labial to lingual aspect[3,4]. Anterior teeth trauma of a young patient is a tragic experience, which requires immediate attention not only because of damage to dentition but also because of the psychological impact it may have on the patient and parents. Various methods and techniques have been employed to restore fractured teeth which include pin retained resin restorations ,orthodontic bands, stainless steel crowns, porcelain jacket crowns, and complex ceramic restorations [5]. However all these restorations require significant tooth preparation and were not esthetically adequate; moreover they cannot be used in an emergency esthetic situation. Reattachment of a fractured fragment when available, can provide esthetically pleasing results. Several studies have reported successful reattachment with long survival time. This case report describes reattachment of a tooth fragment of maxillary central incisors in a thirteen year old girl with coronal fracture involving pulp following trauma

\section{Case Report}

A 13 year old girl reported to the Department of Pedodontics and Preventive Dentistry of the college for treatment of fractured maxillary central incisors. History revealed trauma five days back, medical history was non-contributory. Intra-oral clinical examination revealed a Ellis Class III fracture of maxillary right central incisor, and Ellis Class II fracture of the maxillary left central incisor.(Figure 1) The fractured crown fragment of 21 was brought along by the patient stored in water.(Figure 2) There was no other injury associated with the soft tissues or alveolar bone. Peri-apical radiographs revealed that there was no associated root fracture and the apices were fully formed. The fracture line was located above the gingivo epithelial junction.

Endodontic treatment was carried out for the right maxillary central incisor. The fractured crown fragment was stored in normal saline which was changed weekly to prevent dehydration. Root canal obturation was done using gutta percha. The patient reported one week after obturation for fragment reattachment. The crown fragment was checked for its adaptation and proper alignment on the crown structure.(Figure 3,4) A circumferential V - shaped notch was placed internally in the enamel of the teeth fragments and the teeth. Phosphoric acid gel $37.5 \%$ was applied to the enamel on the fragment and the teeth for 20 seconds, limited to 2 $\mathrm{mm}$ beyond the fracture margin. Bonding Agent was applied to the conditioned areas but not cured at that time. After proper alignment of the crown fragment on the tooth structure, light polymerization of the bonding agent 
was carried out. A small increment of resin composite was applied to the tooth fragment which was then reattached to its proper position. Visible light polymerization was done for 90 seconds while fragment was kept in position under pressure. The teeth were polished with polishing discs. Occlusion was checked and post operative instructions to the patient were given to deter from loading the anterior teeth. (Figure 5,6,7) Clinical and radiographic examination were carried out after 1 month and 3 months, and it was observed that both endodontic and restorative treatments remained clinically acceptable for the entire time.

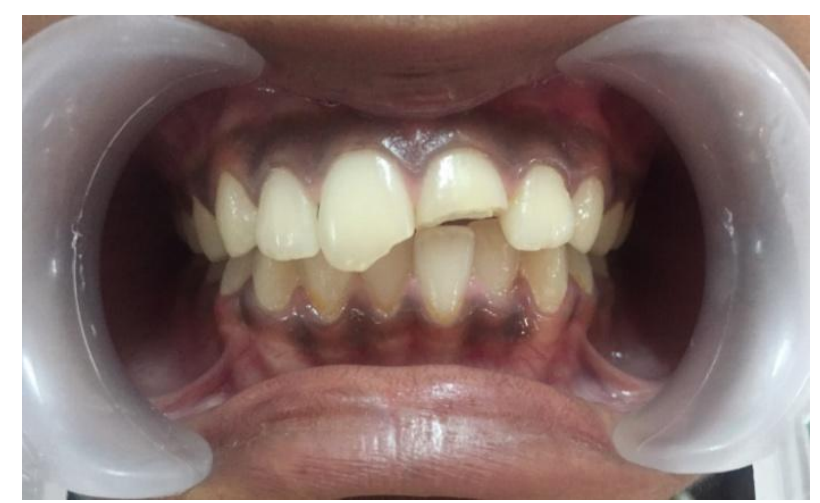

Figure 1. : Ellis Class III fracture of maxillary right central incisor, and Ellis Class II fracture of the maxillary left central incisor

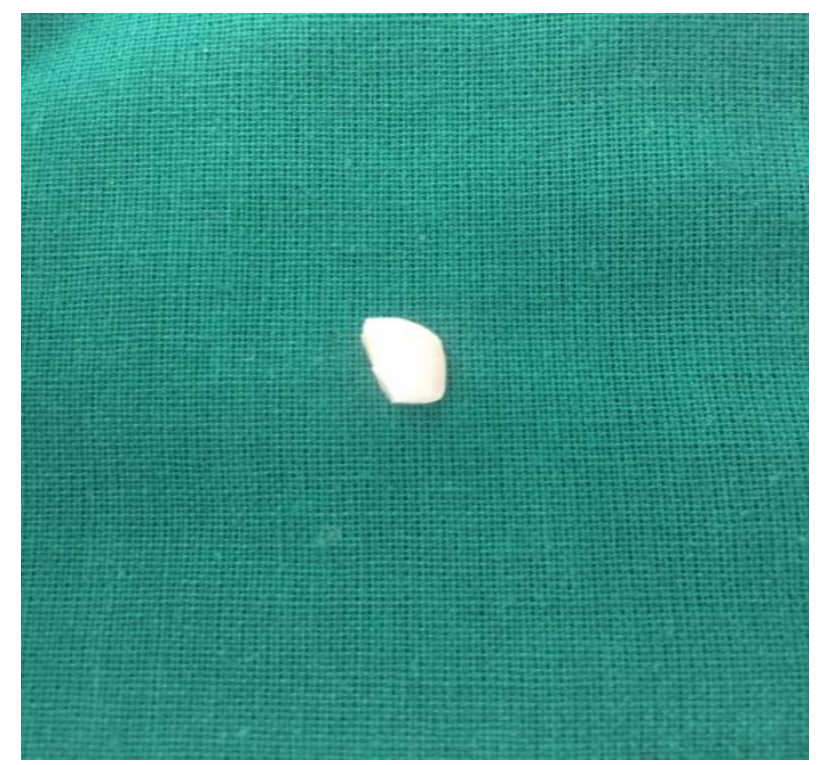

Figure 2. : The fractured crown fragment of 21

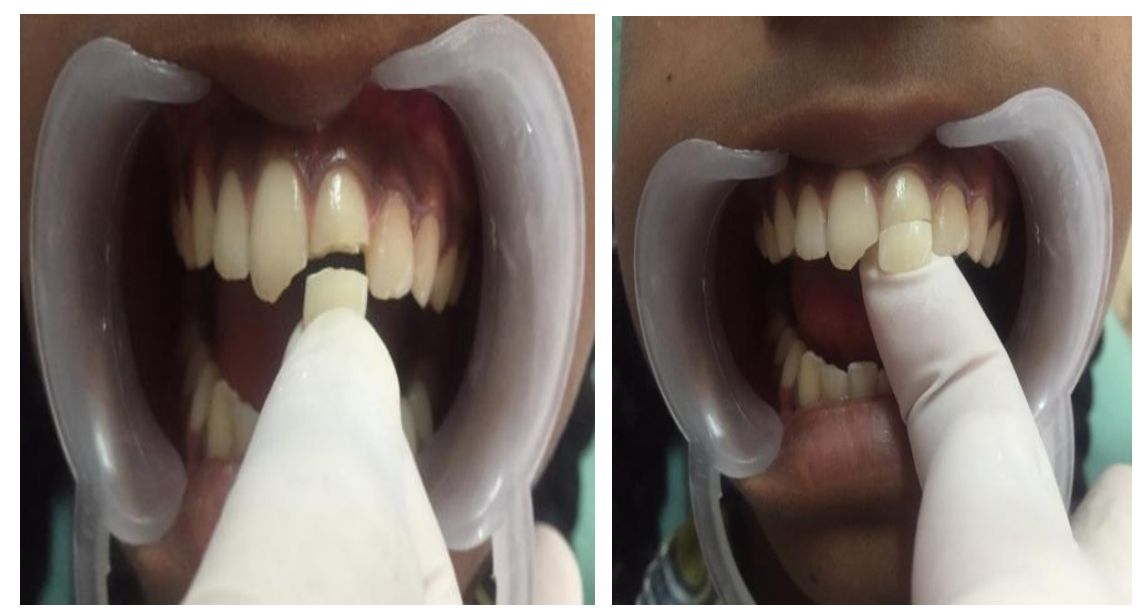

Figure 3,4. : Crown fragment being checked for its adaptation and proper alignment on the crown structure 

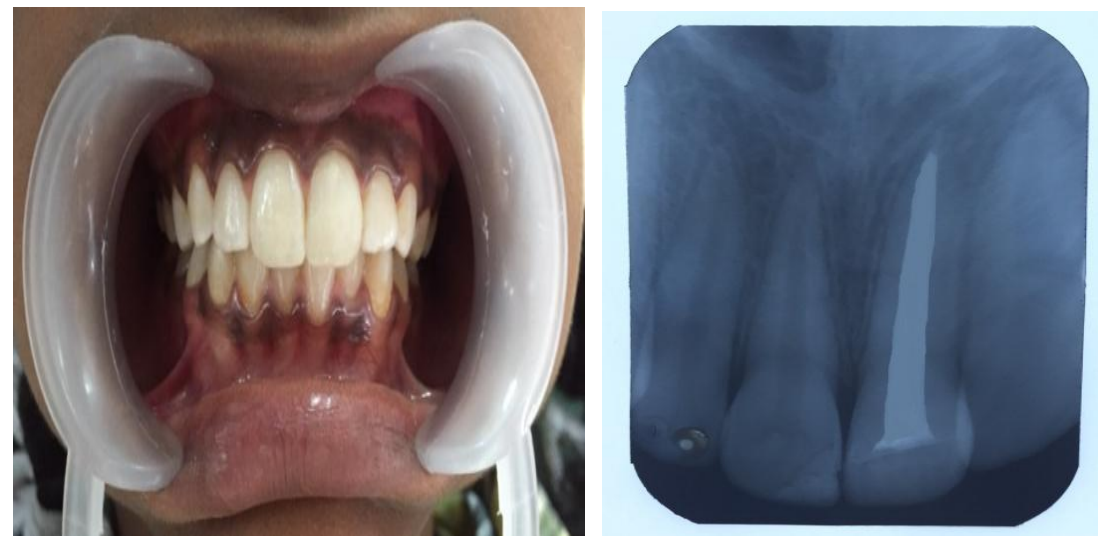

Figure 5, 6. : Post operative intra oral viwe and Intra oral Periapical radiograph

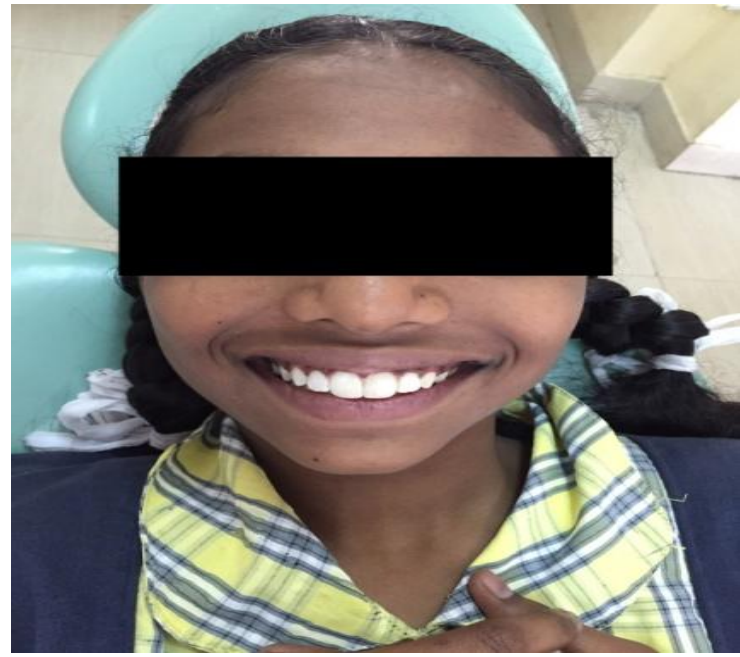

Figure 7. : Post Operative view

\section{Discussion}

The fracture of a tooth may be a most traumatic incident for a young patient, but it has been found that there is a positive emotional and social response from the patient to the preservation of natural tooth structure.[6] The remarkable advancement of adhesive systems and resin composites has made reattachment of tooth fragments a procedure that is no longer a provisional restoration, but rather a restorative treatment which offers a favorable prognosis. However, this technique can be used only when the intact tooth fragment is available.

The first case report on reattachment of a fractured incisor fragment was published by Chosack and Eidelman in 1964 in which the complicated tooth fracture was managed by endodontic therapy followed by a cast post and core. The use of acid etch technique for the reattachment of fractured fragment was first reported by Tennery. Similar cases were also reported by Starkey and Simonsen $[4,5,6]$. The success of reattachment depends on certain factors like the site of fracture, size of fractured remnants, periodontal status, pulpal involvement, maturity of the root formation, biological width invasion, occlusion, time, material used for reattachment, use of posts, and prognosis[7].

Bonding of the crown fragment is a logical restorative treatment option when the tooth fracture results in no or minimal violation of the biological width and when the crown fragment:

(1) is retrieved following the trauma;

(2) is relatively intact; and

(3) adapts well to the remaining tooth.

The reattachment technique promotes immediate positive emotional patient response, excellent esthetics, re-establishment of the tooth's natural surface texture and contours, and adequate shade match when the fragment is not excessively dehydrated. When the tooth fragment is rebonded properly, it also affords adequate occlusal function and wear-resistance. When compared to alternative treatment options, such as direct 
restoration with resin-based composites or indirect restoration with a full-coverage crown, tooth fragment reattachment is a more conservative, affordable, and less time-consuming treatment option.[8]

There are no studies comparing the fracture strength of teeth rebonded with a tooth fragment versus that of teeth restored with a full-coverage restoration; however, it is expected that the rebonded fractured tooth will not be as strong as the intact natural tooth or full coverage restorations. Additionally, the fracture line may become visible over time due to discoloration of the adhesive and composite used for the reattachment.[8]

\section{Conclusion}

Reattachment of the tooth fragment is a way to restore the natural shape, contour, translucency and surface texture of the tooth along with a positive emotional and social response from the patient to the preservation of natural tooth structure. It is also an economical and a conservative procedure. The reattachment of the tooth fragment is possible only when the fragment is available and can be improved with different adhesive techniques and restorative materials. The main concern is to educate the population to preserve the fractured fragment post trauma and seek immediate dental care.

\section{References}

[1]. American Academy of Pediatric Dentistry Council on Clinical Affairs, Guidelines on management of acute dental trauma, Pediatric Dentistry,vol.30,pp.175-183,2008-2009

[2]. C. M. Forsberg and G. Tedestam, Etiological and predisposing factors related to traumatic injuries to permanent teeth, Swedish Dental Journal,vol.17,no.5,pp.183-190,1993.

[3]. A. Reis, A. D. Loguercio, A. Kraul, and E. Matson, Reattachment of fractured teeth: a review of literature regarding techniques and materials,OperativeDentistry,vol.29,no.2,pp. 226-233,2004

[4]. R.J.Simonsen, Restoration of a fractured central incisor using original tooth fragment, The Journal of the American Dental Association, vol.105, no.4, pp.646-648,1982.

[5]. B.Vishwanath, UmranaFaizudin, M.Jayadev, and SushmaShravani, Reattachment of Coronal Tooth Fragment: Regaining Back to Normal. Case Reports in Dentistry Volume2013

[6]. Baratieri L.N., Monteiro S.: Tooth fracture reattachment: Case reports. Quint Int 1990; $21: 261-270$.

[7]. C. P. K. Wadhwani, A single visit, multidisciplinary approach to the management of traumatictooth crown fracture, British Dental Journal, vol.188,no.11,pp.593-598,2000.

[8]. Georgia V. Macedo, André V. Ritter. Essentials of Rebonding Tooth Fragments for the Best Functional and Esthetic Outcomes Pediatr Dent 2009;31:110-6 